\title{
OPTIMAL ACOUSTIC MEASUREMENTS
}

\author{
Margaret Cheney ${ }^{*} \quad$ David Isaacson ${ }^{\dagger} \quad$ Matti Lassas ${ }^{\ddagger}$
}

February 22, 2019

\begin{abstract}
We consider the problem of obtaining information about an inaccessible half-space from acoustic measurements made in the accessible half-space. If the measurements are of limited precision, some scatterers will be undetectable because their scattered fields are below the precision of the measuring instrument. How can we make measurements that are optimal for detecting the presence of an object? In other words, what incident fields should we apply that will result in the biggest measurements?

There are many ways to formulate this question, depending on the measuring instruments. In this paper we consider a formulation involving wavesplitting in the accessible half-space: what downgoing wave will result in an upgoing wave of greatest energy?

A closely related question arises in the case when we have a guess about the configuration of the inaccessible half-space. What measurements should we make to determine whether our guess is accurate? In this case we compare the scattered field to the field computed from the guessed configuration. Again we look for the incident field that results in the greatest energy difference.

We show that the optimal incident field can be found by an iterative process involving time reversal "mirrors". For band-limited incident fields and compactly supported scatterers, in the generic case this iterative process converges to a single time-harmonic field. In particular, the process automatically "tunes" to the best frequency. This analysis provides a theoretical foundation for the frequency-shifting and pulse-broadening observed in certain computations [3] and time-reversal experiments [14] [15].
\end{abstract}

*Department of Mathematical Sciences, Rensselaer Polytechnic Institute, Troy, NY 12180

${ }^{\dagger}$ Department of Mathematical Sciences, Rensselaer Polytechnic Institute, Troy, NY 12180

${ }^{\ddagger}$ Department of Mathematics, P.O. Box 4, 00014 University of Helsinki, FINLAND 


\section{Introduction}

This paper is motivated by the question "What is the best way to do acoustic imaging?" If we want to make the best possible images, we must begin with data that contain the most possible information. In particular, since all practical measurements are of limited precision, some scatterers may be undetectable because their scattered fields are below the precision of the measuring instrument: our data will contain no information about them. What incident fields are "best", in the sense that their scattered fields give the biggest measurements?

This paper considers only the problem of detecting the presence of an object (or distinguishing it from a guess) and not the problem of making an image of that object. For imaging, there are other criteria for "best" that one could imagine using. A Bayesian criterion [9] [10], for example, would be to look for the measurement producing the "narrowest" posterior distribution for the scatterer when an a priori distribution for the scatterer is given.

The detection or distinguishability problem has been studied for fixed-frequency problems in electrical impedance tomography [6] and acoustic scattering [12]. The connection between optimal measurements and iterative time-reversal experiments was pointed out in [12], [14], and [15]; in all these papers, the analysis was carried out at a single fixed frequency. The issue of optimal time-dependent waveforms in a special $1+1$ - dimensional case was studied in [3], where a time-harmonic waveform was found to be optimal.

In this paper we study the question of optimal time-dependent waveforms in the $3+1$ - dimensional case. In particular, we consider the half-space geometry: we imagine that a plane divides space into accessible and inaccessible regions, and we assume that we can make measurements everywhere on the plane.

Section 2 contains a careful formulation of the idealized problem: the wave equation, the measurements, the notion of "biggest". Section 3 is devoted to the example of a one-dimensional medium, in which the problem can be solved explicitly. Section 4 gives an iterative experimental method that can be used to find the optimal field even if the scatterer is unknown. This method is precisely the iterative time-reversal procedure of [14 and [15]. Section 5 discusses implications and open questions. The paper concludes with three appendices containing the technical details needed for the proof of convergence of the time-reversal iterates. We show that in general the iterates converge to a time-harmonic field that is "tuned" to the best frequency.

\section{Basic Concepts}




\subsection{Distinguishability}

For any two operators $A_{1}$ and $A_{2}$, we say that $A_{1}$ is distinguishable from $A_{2}$ with measurement precision $\epsilon$ if the distinguishability $\delta\left(A_{1}, A_{2}\right)$, defined as

$$
\delta\left(A_{1}, A_{2}\right)=\sup _{f} \frac{\left\|A_{1} f-A_{2} f\right\|}{\|f\|},
$$

is greater than $\epsilon$. A field that is best for distinguishing $A_{1}$ from $A_{2}$ is an $f$ for which

the maximum is attained. We will determine below the norms that are appropriate to use.

\subsection{Acoustic Wave Equation}

We consider the constant-density acoustic wave equation

$$
\left(\nabla^{2}-c^{-2}(x) \partial_{t}^{2}\right) U(t, x)=0
$$

in the case in which $c=c_{0}$ everywhere in the upper half-space $x_{3}>0$. This model includes neither dispersion nor dissipation.

We can formulate the scattering problem in a variety of ways [2]. In particular, we can use either a boundary map, sources, or a scattering operator defined in terms of wave splitting.

\subsection{The Boundary Map}

To define the boundary map, we specify that $U=f$ on the surface $x_{3}=0$. This condition, together with an outgoing radiation condition at infinity [2], uniquely determines a solution $U$ in the lower half-space. We can then take the normal derivative $\partial U / \partial x_{3}$; this normal derivative, restricted to the surface $x_{3}=0$, we denote by $g$. The mapping from $f$ to $g$ is the boundary map $\Lambda$. Thus, on the surface $x_{3}=0, \Lambda U=\partial U / \partial x_{3}$. Note that $\Lambda$ is an operator-valued function of time.

Acoustic distinguishability can be defined in terms of the boundary map as

$$
\delta_{B}\left(c, c^{0}\right)=\sup _{f} \frac{\left\|\left(\Lambda-\Lambda_{0}\right) f\right\|}{\|f\|}
$$

for appropriate norms. Here $\Lambda_{0}$ denotes the boundary map for the reference sound speed $c^{0}(x)$. This formulation, in terms of the boundary map, is not pursued in this paper. 


\subsection{Sources}

To formulate scattering in terms of sources, we consider the wave equation with a source:

$$
\left(\nabla^{2}-c^{-2}(x) \partial_{t}^{2}\right) U_{J}(t, x)=J(t, x) .
$$

Scattering data is then $U_{J}(t, x)$ for $x$ on the plane, where $J$ is supported on or above the plane. Acoustic distinguishability in terms of sources would be

$$
\delta_{S}\left(c, c^{0}\right)=\sup _{J} \frac{\left\|U_{J}-U_{J}^{0}\right\|}{\|J\|},
$$

where $U_{J}^{0}$ denotes the field due to the reference sound speed $c^{0}(x)$ and source $J$. This formulation is not pursued in this paper; instead we consider the scattering operator.

\subsection{The Scattering Operator}

We define the scattering operator in terms of upgoing and downgoing waves. The motivation for this point of view is the existence of network analyzers, which can decompose a time-harmonic signal in a waveguide into an upgoing one and a downgoing one, and measure the amplitude and phase of the upgoing wave. Stepped-frequency radar, for example, is based on the ability of such instruments to transmit and receive signals at the same time.

\subsubsection{Upgoing and downgoing waves}

To define upgoing and downgoing waves, we make use of two Fourier transforms, a temporal one and a spatial one. First we inverse-Fourier transform the solution $U$ of (2) in $t$ :

$$
u(\omega, x)=\mathcal{F}^{-1} U=(2 \pi)^{-1} \int U(t, x) e^{i \omega t} d t .
$$

This frequency-domain solution $u$ satisfies the reduced wave equation

$$
\left(\nabla^{2}+\omega^{2} c^{-2}\right) u(\omega, x)=0
$$

We write $k=\omega / c_{0}$ and with a small abuse of notation we write $u(k, x)$ instead of $u\left(c_{0} k, x\right)$. We then Fourier transform $u$ again in $x^{\prime}=\left(x_{1}, x_{2}\right)$, so that

$$
\hat{u}\left(k, \eta^{\prime}, x_{3}\right)=F_{x_{3}} u=\int u(k, x) e^{-i k \eta^{\prime} \cdot x^{\prime}} d^{2} x^{\prime}
$$

where $\eta^{\prime}=\left(\eta_{1}, \eta_{2}\right)$. (We note that $F_{x_{3}}$ depends also on $k$.) Then $U$ is recovered as

$$
U(t, x)=\frac{1}{(2 \pi)^{2}} \iint \hat{u}\left(k, \eta^{\prime}, x_{3}\right) e^{i k \eta^{\prime} \cdot x^{\prime}} e^{-i k c_{0} t} k^{2} d^{2} \eta^{\prime} c_{0} d k .
$$


In the upper half-space, $\hat{u}$ satisfies the ordinary differential equation

$$
\left(\partial_{x_{3}}^{2}+k^{2}-k^{2}\left|\eta^{\prime}\right|^{2}\right) \hat{u}=0,
$$

which has the general solution

$$
\hat{u}\left(k, \eta^{\prime}, x_{3}\right)=A\left(k, \eta^{\prime}\right) e^{i k \eta_{3} x_{3}}+B\left(k, \eta^{\prime}\right) e^{-i k \eta_{3} x_{3}},
$$

where

$$
\eta_{3}(k)= \begin{cases}\sqrt{1-\left|\eta^{\prime}\right|^{2}} & \text { for } 1>\left|\eta^{\prime}\right| \\ i(\operatorname{sgn} k) \sqrt{\left|\eta^{\prime}\right|^{2}-1} & \text { for } 1<\left|\eta^{\prime}\right|\end{cases}
$$

We define the vectors $\eta^{ \pm}=\left(\eta^{\prime}, \pm \eta_{3}\right)$, which satisfy $\eta^{ \pm} \cdot \eta^{ \pm}=1$.

In order for $U$, as defined by (9), to be real-valued, the Fourier transform $\hat{u}$ must satisfy certain symmetry conditions. In particular, we must have $A\left(-k, \eta^{\prime}\right)=\overline{A\left(k, \eta^{\prime}\right)}$, $B\left(-k, \eta^{\prime}\right)=\overline{B\left(k, \eta^{\prime}\right)}$, and $\eta_{3}(-k)=\overline{\eta_{3}(k)}$.

Equation (11) shows us how to split the time-domain solution of (21) into two parts, which we call the upgoing and downgoing parts. Thus for $x_{3}>0$ we write $U=U^{\uparrow}+U^{\downarrow}$, where $U^{\downarrow}$ is

$$
U^{\uparrow}(t, x)=\iint A\left(k, \eta^{\prime}\right) e^{i k \eta^{+} \cdot x} e^{-i k c_{0} t} k^{2} d^{2} \eta^{\prime} c_{0} d k,
$$

and

$$
U^{\downarrow}(t, x)=\iint B\left(k, \eta^{\prime}\right) e^{i k \eta^{-} \cdot x} e^{-i k c_{0} t} k^{2} d^{2} \eta^{\prime} c_{0} d k .
$$

We see that (14) and (13) are plane wave decompositions. The components for which $\left|\eta^{\prime}\right|<1$ are propagating plane waves, and $\eta^{ \pm}$is a unit vector that gives the direction of propagation. The sign of the third component of $\eta^{ \pm}$determines whether the wave is downgoing or upgoing. On the other hand, components with $\left|\eta^{\prime}\right|>1$ correspond to evanescent waves. For $U^{\downarrow}$, these evanescent waves decay in the downward (negative $x_{3}$ ) direction; for $U^{\uparrow}$, they decay in the upward (positive) direction.

\subsubsection{The scattering operator}

It is natural to define a scattering operator $\mathcal{S}^{\downarrow}$ as the map from $U^{\downarrow}$ to $U^{\uparrow}$. We denote the kernel of this operator also by $\mathcal{S} \uparrow$ :

$$
U^{\uparrow}(x, t)=\int_{-\infty}^{\infty} \int \mathcal{S}^{\downarrow}(x, y, t-\tau) U^{\downarrow}(y, \tau) d^{3} y d \tau .
$$

We note that this scattering operator is defined only on downgoing solutions of the Helmholtz equation, i.e., on functions of the form (14). 
The kernel of (15) is a convolution in time because the Fourier transform "diagonalizes" the time derivative of (2), so that the frequency is simply a parameter in (7). In other words, the convolution is an expression of the fact that in the frequency domain, (15) takes the form

$$
u^{\uparrow}(k, x)=\int S^{\downarrow}(k, x, y) u^{\downarrow}(k, y) d^{3} y .
$$

The time-domain operator $\mathcal{S}^{\mathfrak{}}$ is related to the frequency-domain scattering operator $S^{\uparrow}$ by $S^{\uparrow}=\mathcal{F}^{-1} \mathcal{S} \mathcal{F}$.

In fact, $\mathcal{S}^{\downarrow}$ and $S^{\uparrow}$ are determined by their actions on the plane $x_{3}=0$. We see this as follows. First we Fourier transform (16) in space. The operator $S^{\uparrow}$ is transformed into the operator $\hat{S}_{x_{3}, \tilde{x}_{3}}=F_{x_{3}} S^{\uparrow} F_{\tilde{x}_{3}}^{-1}=\left(F_{x_{3}} \mathcal{F}^{-1}\right) \mathcal{S}^{\ddagger}\left(F_{\tilde{x}_{3}} \mathcal{F}^{-1}\right)^{-1}$, where $F_{x_{3}}$ is defined by (8) and $\mathcal{F}^{-1}$ by (6). The transformed version of (16) is

$$
\hat{u}^{\uparrow}\left(k, \eta^{\prime}, x_{3}\right)=\int \hat{S}_{x_{3}, \tilde{x}_{3}}\left(k, \eta^{\prime}, \tilde{\eta}^{\prime}\right) \hat{u}^{\downarrow}\left(k, \tilde{\eta}^{\prime}, \tilde{x}_{3}\right) k^{2} d^{2} \tilde{\eta}^{\prime}
$$

The operator $\hat{S}_{x_{3}, \tilde{x}_{3}}$ is determined completely by its action at $x_{3}=0$, which we see from the following argument.

Into (17) we substitute $\hat{u}^{\downarrow}\left(k, \tilde{\eta}^{\prime}, \tilde{x}_{3}\right)=B\left(k, \tilde{\eta}^{\prime}\right) e^{-i k \tilde{\eta}_{3} \tilde{x}_{3}}$ and $\hat{u}^{\uparrow}\left(k, \eta^{\prime}, x_{3}\right)=A\left(k, \eta^{\prime}\right) e^{i k \eta_{3} x_{3}}$; we see that

$$
\hat{S}\left(k, \eta^{\prime}, \tilde{\eta}^{\prime}\right)=e^{-i k \eta_{3} x_{3}} \hat{S}_{x_{3}, \tilde{x}_{3}}\left(k, \eta^{\prime}, \tilde{\eta}^{\prime}\right) e^{-i k \tilde{\eta}_{3} \tilde{x}_{3}}
$$

satisfies

$$
A\left(k, \eta^{\prime}\right)=\int \hat{S}\left(k, \eta^{\prime}, \tilde{\eta}^{\prime}\right) B\left(k, \tilde{\eta}^{\prime}\right) k^{2} d^{2} \tilde{\eta}^{\prime}
$$

where $A$ and $B$ are as in (11). The relation between the operators $\hat{S}$ and $\hat{S}_{x_{3}, \tilde{x}_{3}}$ can be written $\hat{S}_{x_{3}, \tilde{x}_{3}}=E_{x_{3}} \hat{S} E_{\tilde{x}_{3}}$, where $E_{x_{3}}$ is the operator of multiplication by $\exp \left(i k \eta_{3} x_{3}\right)$, and thus

$$
S^{\uparrow}=F_{x_{3}}^{-1} E_{x_{3}} \hat{S} E_{\tilde{x}_{3}} F_{\tilde{x}_{3}} .
$$

On the plane $x_{3}=0$, this becomes

$$
S=F_{0}^{-1} \hat{S} F_{0}
$$

This defines a scattering operator $S$ on the plane $x_{3}=0$. It is this operator, together with the corresponding time-domain operator $\mathcal{S}=\mathcal{F} S \mathcal{F}^{-1}$, that we will use in the rest of the paper. We note that the domain of the operator $\mathcal{S}$ is restricted to the space of downgoing waves as defined by (14).

The scattering operator $\hat{S}$ and the boundary map $\Lambda$ are related to each other by formulas developed in [2] (See Appendix A for details). They are thus equivalent operators. Whether it is better to formulate a given problem in terms of a scattering operator or a boundary map depends largely on the design of the equipment involved. 


\subsection{The Energy Identity and the Energy Flux}

If we multiply (2) by $\partial_{t} U$ and integrate the resulting equation over the volume $V$, we obtain

$$
\int_{V}\left(\left(\partial_{t} U\right) \nabla^{2} U-\frac{1}{2 c^{2}(x)} \partial_{t}\left(\partial_{t} U\right)^{2}\right) d x=0 .
$$

We write the first term of (22) as $\nabla \cdot\left(\left(\partial_{t} U\right) \nabla U\right)-\nabla\left(\partial_{t} U\right) \cdot \nabla U$, and apply the divergence theorem to the term containing the divergence. We thus obtain

$$
\int_{\partial V}\left(\partial_{t} U\right) \partial_{\nu} U d S=\partial_{t} \int_{V} \frac{1}{2}\left(|\nabla U|^{2}+\frac{1}{c^{2}(x)}\left(\partial_{t} U\right)^{2}\right) d x
$$

where $\nu$ denotes the outward unit normal to the surface $\partial V$.

This equation relates the change in energy in the volume $V$ (the right side of (23)) to the energy flux across its boundary surface $\partial V$.

From (23) we see that the time-integrated energy flux across a surface $\partial V$ in the normal direction $\nu$ is

$$
W(U)=-\int_{-\infty}^{\infty} \int_{\partial V}\left(\partial_{t} U\right) \partial_{\nu} U d S d t
$$

We can use Parseval's identity to write the time-integrated energy flux in terms of the frequency-domain wave functions:

$$
W(u)=-(2 \pi)^{3} \int_{-\infty}^{\infty} \int_{\partial V}(i \omega u) \overline{\partial_{\nu} u} d S d \omega=-(2 \pi)^{3} \int_{-\infty}^{\infty} \int_{\partial V}\left(i c_{0} k u\right) \overline{\partial_{\nu} u} d S c_{0} d k,
$$

where the overline denotes the complex conjugate.

\subsubsection{The energy flux of upgoing and downgoing waves}

At the surface $x_{3}=0$, the total field splits into upgoing and downgoing parts. Because the flux is quadratic, it does not necessarily split into corresponding upgoing and downgoing fluxes. However, a quick calculation using (9), (11), and the Fourier inversion formula shows that the time-integrated cross terms $\int_{-\infty}^{\infty} \int_{x_{3}=0}\left(\partial_{t} U^{\downarrow}\right) \partial_{x_{3}} U^{\uparrow} d x^{\prime} d t$ and $\int_{-\infty}^{\infty} \int_{x_{3}=0}\left(\partial_{t} U^{\uparrow}\right) \partial_{x_{3}} U^{\downarrow} d x^{\prime} d t$ cancel. This shows that the time-integrated fluxes do split into upgoing and downgoing fluxes, so that $W\left(U^{\downarrow}+U^{\uparrow}\right)=W\left(U^{\downarrow}\right)+W\left(U^{\uparrow}\right)$. The flux of a downgoing wave is positive; that of an upgoing wave is negative.

We write the downgoing energy flux as

$$
W\left(U^{\downarrow}\right)=\int_{-\infty}^{\infty} \int_{x_{3}=0}\left(\partial_{t} U^{\downarrow}\right) \partial_{x_{3}} U^{\downarrow} d x^{\prime} d t=(2 \pi)^{3} \int_{-\infty}^{\infty} \int\left|B\left(k, \eta^{\prime}\right)\right|^{2} c_{0}^{2} \eta_{3} k^{4} d^{2} \eta^{\prime} d k
$$

where we have used (14) and (25) in carrying out the computation (26). In (26) there is no minus sign because downgoing energy travels in the $-x_{3}$ direction. 
Although the left side of (26) is real, it is not obvious that the right side is, because $\eta_{3}$ can be imaginary. However, if one splits the $k$ integral into pieces as

$$
W\left(U^{\downarrow}\right)=\int\left(\int_{-\infty}^{0}+\int_{0}^{\infty}\right)\left|B\left(k, \eta^{\prime}\right)\right|^{2} c_{0}^{2} k^{4} \eta_{3} d k d^{2} \eta^{\prime}
$$

and uses the symmetry properties of $B$ and $\eta_{3}$, one sees that for evanescent waves, the two terms cancel. This shows that the evanescent waves do not contribute to the energy flux.

The flux can be used to form an inner product on the space of downgoing propagating waves; we define

$$
\begin{aligned}
\left(U^{\downarrow}, V^{\downarrow}\right)_{W} & =\frac{1}{2} \int_{-\infty}^{\infty} \int_{x_{3}=0}\left(\left(\partial_{t} U^{\downarrow}\right) \partial_{x_{3}} V^{\downarrow}+\left(\partial_{t} V^{\downarrow}\right) \partial_{x_{3}} U^{\downarrow}\right) d^{2} x^{\prime} d t \\
& =(2 \pi)^{3} \iint_{\left|\eta^{\prime}\right|<1} \hat{u}^{\downarrow} \hat{v}^{\downarrow} c_{0}^{2} k^{4} \eta_{3} d k d^{2} \eta^{\prime}
\end{aligned}
$$

We note that the product $c_{0}^{2} k^{4} \eta_{3}$ is non-negative, so in the transform domain, this inner product is merely a weighted $L^{2}$ inner product.

Similarly, the energy flux of the upgoing scattered field $S U^{\downarrow}=U^{\uparrow}$ that passes through the plane $x_{3}=0$ is

$$
W\left(U^{\uparrow}\right)=\int_{-\infty}^{\infty} \int_{x_{3}=0}\left(\partial_{t} U^{\uparrow}\right) \partial_{x_{3}} U^{\uparrow} d x^{\prime} d t=-(2 \pi)^{3} \int_{-\infty}^{\infty} \int\left|A\left(k, \eta^{\prime}\right)\right|^{2} c_{0}^{2} k^{4} \eta_{3} d^{2} \eta^{\prime} d k .
$$

The minus sign in (29) is due to the fact that the upgoing wave corresponds to energy leaving the lower half-space. Equations (26) and (29) show that the time-integrated downgoing flux $W\left(U^{\downarrow}\right)$ is positive and the time-integrated upgoing flux $W\left(U^{\uparrow}\right)$ is negative.

Note that for propagating waves, $|W|$ satisifes the triangle inequality: $\mid W\left(U_{1}^{\uparrow}+\right.$ $\left.U_{2}^{\uparrow}\right)|\leq| W\left(U_{1}^{\uparrow}\right)|+| W\left(U_{2}^{\uparrow}\right) \mid$.

The flux inner product on the space of upgoing propagating waves is

$$
\begin{aligned}
\left(U^{\uparrow}, V^{\uparrow}\right)_{W} & =\left|\frac{-1}{2} \int_{-\infty}^{\infty} \int_{x_{3}=0}\left(\left(\partial_{t} U^{\uparrow}\right) \partial_{x_{3}} V^{\uparrow}+\left(\partial_{t} V^{\uparrow}\right) \partial_{x_{3}} U^{\uparrow}\right)\right| d^{2} x^{\prime} d t \\
& =(2 \pi)^{3} \iint_{\left|\eta^{\prime}\right|<1} \hat{u}^{\uparrow} \overline{\hat{v}^{\uparrow}} c_{0}^{2} k^{4} \eta_{3} d k d^{2} \eta^{\prime}
\end{aligned}
$$

thus for propagating waves, $\left|W\left(U^{\uparrow}\right)\right|=\left(U^{\uparrow}, U^{\uparrow}\right)_{W}$.

Conservation of energy. If the medium is initially quiescent, conservation of energy tells us that $W\left(U^{\downarrow}\right) \geq\left|W\left(\mathcal{S} U^{\downarrow}\right)\right|$; this can be seen from integrating (23) over all time, and using the fact that the energy within the volume $V$ is initially zero, and cannot become negative. The time integral of the right side of (23) is thus positive. The left side we write as $W(U)=W\left(U^{\downarrow}\right)+W\left(\mathcal{S} U^{\downarrow}\right)$. This implies that the total upgoing flux $\left|W\left(\mathcal{S} U^{\downarrow}\right)\right|$ cannot be greater than the total downgoing flux $W\left(U^{\downarrow}\right)$. 
Finite-energy fields on the plane. We define the space $w$ of finite-energy functions on the plane to be the closure of $C_{0}^{\infty}\left(\mathbf{R}^{2} \times \mathbf{R}\right)$ in the inner product

$$
(u, v)_{w}^{2}=\iint \hat{u}(k, \eta) \overline{\hat{v}(k, \eta)} c_{0}^{2} k^{4}\left|\eta_{3}\right| d^{2} \eta^{\prime} d k
$$

and the space $W=\mathcal{F} w$.

\subsection{Acoustic Distinguishability via the Scattering Operator}

We define the acoustic distinguishability in terms of the upgoing and downgoing energy fluxes through the surface $x_{3}=0$.

For a reference scatterer with scattering operator $\mathcal{S}_{0}$, the energy flux of the upgoing scattered field $\mathcal{S}_{0} U^{\downarrow}$ and of the difference field $\left(\mathcal{S}-\mathcal{S}_{0}\right) U^{\downarrow}$ are defined similarly.

In general the distinguishability of $\mathcal{S}$ from $\mathcal{S}_{0}$ with the incident field $U^{\downarrow}$ is

$$
\delta\left(\mathcal{S}, \mathcal{S}_{0}\right)=\sup _{U^{\downarrow}} \frac{\left|W\left(\left(\mathcal{S}-\mathcal{S}_{0}\right) U^{\downarrow}\right)\right|}{W\left(U^{\downarrow}\right)}=\sup _{u^{\downarrow}} \frac{\left|W\left(\left(S-S_{0}\right) u^{\downarrow}\right)\right|}{W\left(u^{\downarrow}\right)} .
$$

We recall that evanescent components do not contribute to the energy flux. To remove the evanescent components from (32), we denote by $P$ the orthogonal projection onto the propagating components: $P=F_{0}^{-1} \hat{P} F_{0}$, where $\hat{P}$ is the operator of multiplication by $\chi_{\left|\eta^{\prime}\right|<1}$, the function that is one for $\left|\eta^{\prime}\right|<1$ and zero otherwise. Explicitly, $P$ is given by

$$
P(k) f\left(x^{\prime}\right)=\int k^{2} \int_{\left|\eta^{\prime}\right|<1} e^{i k \eta^{\prime} \cdot\left(x^{\prime}-y^{\prime}\right)} d^{2} \eta^{\prime} f\left(y^{\prime}\right) d^{2} y^{\prime} .
$$

In the time domain, $\mathcal{P}=\mathcal{F} P \mathcal{F}^{-1}$. With this notation, we can write $W(U)=$ $W(\mathcal{P} U)=W(P u)$.

Moreover, the scattered field due to an evanescent incident wave has zero total energy flux. This is because of the comments at the end of the previous section: $0=W\left((I-P) u^{\downarrow}\right) \geq\left|W\left(S(I-P) u^{\downarrow}\right)\right|$ implies that $W\left(S(I-P) u^{\downarrow}\right)=0$.

In addition, the upgoing energy flux can only be increased by getting rid of the evanescent components of the incident wave. This is because of the triangle inequality $|W(S P u+S(I-P) u)| \leq|W(S P u)|+\mid W(S(I-P) u|=| W(S P u) \mid$.

This implies that the downgoing waves that give rise to the maximum total energy flux are propagating waves. Thus we find that the distinguishability can be written

$$
\delta\left(S, S_{0}\right)=\sup _{U^{\downarrow}} \frac{\left|W\left(\mathcal{P}\left(\mathcal{S}-\mathcal{S}_{0}\right) \mathcal{P} U^{\downarrow}\right)\right|}{W\left(\mathcal{P} U^{\downarrow}\right)}=\sup _{u \downarrow} \frac{\left|W\left(P\left(S-S_{0}\right) P u^{\downarrow}\right)\right|}{W\left(P u^{\downarrow}\right)} .
$$


We note that the scattering operator $\mathcal{S}_{0}$ for free space is the zero operator. Thus, according to (1) and (32), the presence of a scatterer can be detected with measurement precision $\epsilon$ if the distinguishability satisfies

$$
\delta(\mathcal{S}, 0)=\sup _{U^{\downarrow}} \frac{\left|W\left(\mathcal{P} \mathcal{S P} U^{\downarrow}\right)\right|}{W\left(\mathcal{P} U^{\downarrow}\right)}=\sup _{\mathcal{P} U^{\downarrow}} \frac{\left|W\left(\mathcal{P} U^{\uparrow}\right)\right|}{W\left(\mathcal{P} U^{\downarrow}\right)}>\epsilon .
$$

The distinguishability can be defined equally well in terms of the operator $\hat{S}$ of (18) or $S$ of (21).

\section{Example: The One-Dimensional Case}

If the medium in the lower half-space depends only on depth, then the coefficient $B$ of (11) is the reflection coefficient $R\left(k, \eta^{\prime}\right)$ multiplied by the incident coefficient $A$. In this case, the distinguishability $\delta(\mathcal{S}, 0)$ can be computed from (26), (29), and (35) as

$$
\delta(\mathcal{S}, 0)=\sup _{B} \frac{\iint\left|R\left(k, \eta^{\prime}\right) B\left(k, \eta^{\prime}\right)\right|^{2} c_{0}^{2} k^{4} \eta_{3} d k d^{2} \eta^{\prime}}{\iint\left|B\left(k, \eta^{\prime}\right)\right|^{2} c_{0}^{2} k^{4} \eta_{3} d k d^{2} \eta^{\prime}} .
$$

The maximum of the right side of $(36)$ is attained in the limit when $B$ is a delta function supported at the maximum of $|R|$.

Thus to maximize the scattering from a one-dimensional scatterer, we compute the conventional reflection coefficient $R\left(k, \eta^{\prime}\right)$, and find the values of $k$ and $\eta^{\prime}$ at which it attains its maximum. Taking $B$ to be a delta function supported at these points corresponds to taking an incident field that is a plane wave of fixed frequency $\omega=c_{0} k$ and incident direction given by $\eta^{\prime}$.

Note that since $R$ is minus one for $\left|\eta^{\prime}\right|=1$ (grazing), a maximum always occurs at grazing incidence. If this is undesirable, grazing incidence can be excluded by modifying the definition of distinguishability.

\section{An Adaptive Method for Producing the Best Fields}

To maximize the distinguishability when the medium is unknown, we can use the following adaptive method.

We write

$$
\delta\left(\mathcal{S}, \mathcal{S}_{0}\right)=\sup _{U^{\downarrow}} \frac{\left|W\left(\mathcal{P}\left(\mathcal{S}-\mathcal{S}_{0}\right) \mathcal{P} U^{\downarrow}\right)\right|}{W\left(\mathcal{P} U^{\downarrow}\right)}=\sup _{U \in W} \frac{\left(U,\left(\mathcal{P}\left(\mathcal{S}-\mathcal{S}_{0}\right) \mathcal{P}\right)^{*} \mathcal{P}\left(\mathcal{S}-\mathcal{S}_{0}\right) \mathcal{P} U\right)_{W}}{(\mathcal{P} U, \mathcal{P} U)_{W}}
$$


where $U(x, t)=\left.U^{\downarrow}\right|_{x_{3}=0}$ and where the adjoint ${ }^{*}$ has been taken in the space $W$ (defined just below (31)).

We see in Appendix A that $(\mathcal{P S P})^{*}=T(\mathcal{P S P}) T$, where $T$ denotes the timereversal operator $T U(t, x)=U(-t, x)$.

Thus we see that the operator appearing on the right side of (37) is $\mathcal{A}=T \mathcal{P}(\mathcal{S}-$ $\left.\mathcal{S}_{0}\right) \mathcal{P} T\left(\mathcal{S}-\mathcal{S}_{0}\right)$. In general, to maximize a quotient of the form

$$
\langle U, \mathcal{A} U\rangle /\langle U, U\rangle
$$

one considers an appropriately normalized sequence $\mathcal{A}^{n} U$. When $\mathcal{A}$ is compact, this sequence converges to the largest eigenvalue of $\mathcal{A}$. Here, however, $\mathcal{A}$ has a continuous spectrum, so we expect the sequence $\mathcal{A}^{n} U$, when appropriately normalized, to converge to a generalized eigenfunction of $\mathcal{A}$, and the corresponding quotient (38) to converge to the supremum of the continuous spectrum. We note that such generalized eigenfunctions do not have finite energy.

When $\mathcal{A}$ is compact, the usual way to normalize $\mathcal{A}^{n} U$ is to divide by $\left\|\mathcal{A}^{n} U\right\|$. Here, however, because we expect $\mathcal{A}^{n} U$ to converge to a distribution in the time variable, we must use a distributional normalization. We consider test functions in a particular space that is discussed in the appendix. These test functions are functions of space and time. In the time variable, they are Fourier transforms of functions of compact support. The distribution action is chosen to coincide with the flux inner product defined by (28) and (30). For the distribution action we use the same notation $(\cdot, \cdot)_{W}$ as for the flux inner product.

To normalize, we choose an arbitrary test function $\Psi$, and consider the sequence $\mathcal{A}^{n} U /\left(\mathcal{A}^{n} U, \Psi\right)_{W}$. This gives rise to the following algorithm for carrying out the maximization of (37).

1. Start with any $V_{0}^{\downarrow}$; let $j=0$.

2. Send $V_{j}^{\downarrow}$ into the lower half-space, and measure the resulting upgoing field $V_{j}^{\uparrow}(t, x)=\mathcal{S} V_{j}^{\downarrow}(t, x)$.

3. Calculate the corresponding scattering from the reference configuration $\left.\mathcal{S}_{0} V_{j}^{\downarrow}(t, x)\right)$. Calculate the difference field $\tilde{V}_{j}^{\uparrow}(t, x)=V_{j}^{\uparrow}(t, x)-\mathcal{S}_{0} V_{j}^{\downarrow}(t, x)$.

4. If $j$ is even, let

$$
V_{j+1}^{\downarrow}(t, x)=\tilde{V}_{j}^{\uparrow}(-t, x)
$$

add one to $j$, and go to Step 2 .

5. If $j$ is odd, normalize:

$$
V_{j+1}^{\downarrow}(t, x)=\frac{\tilde{V}_{j}^{\uparrow}(-t, x)}{\left(T \tilde{V}_{j}^{\uparrow}, \Psi\right)_{W}},
$$

add one to $j$, and go to Step 2 . 
Appendix B contains a proof that, in the case of a compactly supported scatterer in free space, the sequence $U_{n}=V_{2 n}=\mathcal{A}^{n} U$ generally converges to a single timeharmonic wave. The frequency of this wave is the frequency at which the largest eigenvalue of $S$ attains its maximum. If this largest eigenvalue happens to attain the same maximum at several different frequencies, then the iterates $U_{n}$ converge to a sum of time-harmonic waves with these frequencies. The relative strengths of the different frequencies is determined by the corresponding frequency components of the initial incident wave $U_{0}=V_{0}^{\downarrow}$.

The argument in Appendix B takes place within a limited frequency band; this frequency band is determined by the bandwidth of the test function.

We note that as expected, the limiting time-harmonic waves do not have finite energy. This property also appears in the one-dimensional example (36).

Step 4 can be omited and Step 5 performed for every $j$ : the linearity of the problem implies that extra normalizations do not affect the limit. The proof in the Appendix, however, corresponds to the above algorithm.

The algorithm can also be implemented including a step in which the evanescent waves are filtered out. If they are not filtered out, however, they will die out anyway as the iteration proceeds, because experimental time-reversal of a field that includes evanescent waves is simply another physical field with evanescent waves.

\section{Conclusions and Open Questions}

This analysis shows that the iterative time-reversal work of [14] and [15] provides an experimental method to obtain optimal fields. Moreover, this analysis explains the frequency-shifting and pulse-broadening seen in [15] and [3]: the optimal time-domain waveform is a time-harmonic one tuned to the best frequency.

This analysis suggests that the commonly-used pings and chirps are not optimal from the point of view of distinguishability.

There are many open questions related to this work, one of which is the question of limited-aperture and limited-time measurements. Upgoing and downgoing waves in a limited aperture can be defined with the help of eigenvalues of the Laplacian for the aperture. However, it is not clear how to determine the entire incident wave if the incident wave is known in only a limited aperture. This involves a detailed modeling of the transducer or antenna. Perhaps a formulation in terms of sources will be more useful in this case.

We have not studied the question of whether the distinguishability, as a function of the medium, is monotone in any sense. This is an important issue for the following reason. Suppose we discover that a sphere of a certain radius is detectable with a certain measurement precision. Does this imply that a larger object will also be 
detectable? For fixed-frequency measurements, the answer to this question is certainly no, because of the phenomenon of resonance. A small sphere may happen to have a radius commensurate with the wavelength of the probing wave, and may therefore scatter much more strongly than a larger sphere. However, the use of time-dependent fields may give different results.

The simple wave equation studied in this paper does not include the important effects of variable density, dispersion, and dissipation.

Moreover, the question of distinguishability is only the first step in building an optimal imaging system. How should we choose a full set of optimal fields that could be used to form an image?

\section{Acknowledgments}

This work was partially supported by the Office of Naval Research. M.C. would like to thank a number of people for helpful discussions: Gerhard Kristensson and his group in Lund, Jim Rose, Claire Prada, and Isom Herron; M.L. thanks Lassi Päivärinta for interesting discussions.

\section{A Appendix: Properties of $S$.}

\section{A.1 Expression for Kernel}

We can find an expression for the kernel $\hat{S}$ of (17) and (18) by taking $\hat{u}^{\downarrow}$ to be a delta function. The kernel of $\hat{S}$ is then the corresponding upgoing wave $\hat{u}^{\uparrow}$. Taking $\hat{u}^{\downarrow}$ to be a delta function means that we take $u^{\downarrow}$ of the form $\exp \left(i k \tilde{\eta}^{-} \cdot x\right)$ for some $\tilde{\eta}^{-}=\left(\tilde{\eta}^{\prime},-\tilde{\eta}_{3}\right)$. Here $\tilde{\eta}_{3}$ can be complex. We write the corresponding frequencydomain field as $\psi$ :

$$
\psi\left(k, x, \tilde{\eta}^{-}\right)=\frac{1}{2 \pi} \int U\left(t, x, \tilde{\eta}^{-}\right) e^{i k c_{0} t} d t
$$

so that

$$
\hat{u}\left(k, \eta^{\prime}, x_{3}, \tilde{\eta}^{-}\right)=\int \psi\left(k, x, \tilde{\eta}^{-}\right) e^{-i k \eta^{\prime} \cdot x^{\prime}} d^{2} x^{\prime}
$$

In the case of scattering from a perturbation in free space, we can express the total frequency-domain field $\psi$ as a solution of the Lippmann-Schwinger equation

$$
\psi(k, x, \tilde{\eta})=\exp (i k \tilde{\eta} \cdot x)-k^{2} \int g(k, x, y) V(y) \psi(k, y, \tilde{\eta}) d^{3} y,
$$

where $g$ is the usual outgoing Green's function

$$
g(k, x, y)=\frac{e^{i k|x-y|}}{4 \pi|x-y|}
$$


and $V(y)=1-c_{0}^{2} / c^{2}(y)$. The scattered field $u^{\uparrow}$ thus is represented by the integral term of (43).

The Green's function can be written in terms of its two-dimensional Fourier transform as [2]

$$
g(k, x, y)=\frac{1}{(2 \pi)^{2}} \int \frac{i}{2 k \eta_{3}} e^{i k \eta_{3}\left|x_{3}-y_{3}\right|} e^{i k \eta^{\prime} \cdot\left(x^{\prime}-y^{\prime}\right)} k^{2} d^{2} \eta^{\prime} .
$$

To compute $A$ and $B$ of (11), we take the $x_{1}, x_{2}$ Fourier transform of (43) in the region $x_{3}>0$. We assume that the perturbation $V$ is supported in the region $y_{3}<0$, so that when we consider (43) we can remove the absolute values in (45). In the transform domain, the scattered field is given by

$$
\hat{u}^{\uparrow}\left(k, \eta^{\prime}, x_{3}, \tilde{\eta}^{-}\right)=-k^{2} \int \frac{i}{2 k \eta_{3}} \int e^{-i k \eta^{+} \cdot y} V(y) \psi\left(k, y, \tilde{\eta}^{-}\right) d^{2} y^{\prime} d y_{3} e^{i k \eta_{3} x_{3}} .
$$

This shows that

$$
\hat{S}\left(k, \eta^{\prime}, \tilde{\eta}^{\prime}\right)=-\frac{i k}{2 \eta_{3}} A\left(k, \eta^{+}, \tilde{\eta}^{-}\right)
$$

where

$$
A(k, \eta, \tilde{\eta})=\int e^{-i k \eta \cdot y} V(y) \psi(k, y, \tilde{\eta}) d^{3} y
$$

is a scalar multiple of the classical scattering amplitude 113 . (This $A$ is not to be confused with the $A$ of (11)!) It satisfies the reciprocity relation

$$
A(k, \eta, \tilde{\eta})=A(k,-\tilde{\eta},-\eta)
$$

and the symmetry relation (for real-valued perturbations $V$ )

$$
\overline{A(k, \eta, \tilde{\eta})}=A(-k, \eta, \tilde{\eta})
$$

It is clear from (47) that $\hat{S}$ is an analytic function of $k$ [13].

An expression similar to (47) can be obtained for the field scattered from a perturbed half-space or layered medium; in this case the appropriate background Green's function should be used instead of the free-space Green's function in (43). Scattering theory in such cases is considered, for example, in [19], [20], and [7].

\section{A.2 The Adjoint}

For a scatterer in free space, the adjoint of $\mathcal{P S P}$ in the space $W$ can be computed explicitly as follows. From (30) we have

$$
(\mathcal{P S P} U, V)_{W}=(2 \pi)^{3} \int_{-\infty}^{\infty} \int_{\left|\eta^{\prime}\right|<1} \int_{\left|\tilde{\eta}^{\prime}\right|<1} \hat{S}\left(k, \eta^{\prime}, \tilde{\eta}^{\prime}\right) \hat{u}\left(k, \tilde{\eta}^{\prime}\right) k^{2} d^{2} \tilde{\eta}^{\prime} \overline{\hat{v}\left(k, \eta^{\prime}\right)} c_{0}^{2} k^{4} \eta_{3} d k d^{2} \eta^{\prime}
$$




$$
=(2 \pi)^{3} \int_{-\infty}^{\infty} \int_{\left|\eta^{\prime}\right|<1} \hat{u}\left(k, \tilde{\eta}^{\prime}\right) \overline{\int_{\left|\tilde{\eta}^{\prime}\right|<1} \overline{\hat{S}\left(k, \eta^{\prime}, \tilde{\eta}^{\prime}\right)} \hat{v}\left(k, \eta^{\prime}\right) \eta_{3} d^{2} \eta^{\prime}} c_{0}^{2} k^{6} d k d^{2} \tilde{\eta}^{\prime}
$$

From (47), (49), and (50), we have

$$
\begin{aligned}
\frac{(\mathcal{P S P} U, V)_{W}}{(2 \pi)^{3}} & =\int_{-\infty}^{\infty} \int_{\left|\eta^{\prime}\right|<1} \hat{u}\left(k, \tilde{\eta}^{\prime}\right) \overline{\int_{\left|\tilde{\eta}^{\prime}\right|<1} \overline{-(i k / 2) A\left(k,-\tilde{\eta}^{-},-\eta^{+}\right)} \hat{v}\left(k, \eta^{\prime}\right) d^{2} \eta^{\prime}} c_{0}^{2} k^{6} d k d^{2} \tilde{\eta}^{\prime} \\
& =\int_{-\infty}^{\infty} \int_{\left|\eta^{\prime}\right|<1} \hat{u}\left(k, \tilde{\eta}^{\prime}\right) \\
& =\int_{-\infty}^{\infty} \int_{\left|\tilde{\eta}^{\prime}\right|<1} \hat{u}\left(k, \tilde{\eta}^{\prime}\right)
\end{aligned}
$$

where in the last equality we have used the fact that $\hat{S}\left(-k, \eta^{\prime}, \tilde{\eta}^{\prime}\right)=i k\left(2 \eta_{3}\right)^{-1} A\left(-k, \eta^{+}, \tilde{\eta}^{-}\right)$. We note that in the course of this computation, the $\eta_{3}$ has disappeared and has been replaced by $\tilde{\eta}_{3}$; this is because of the $\eta_{3}$ in the denominator of (47).

We see that the action of the adjoint $(\mathcal{P S P})^{*}$ on $\mathrm{V}$ is given in the transform domain by the $\eta^{\prime}$ integral of (52):

$$
F \mathcal{F}^{-1}(\mathcal{P S P})^{*} \mathcal{F} F^{-1} \hat{v}\left(k, \tilde{\eta}^{\prime}\right)=\int_{\left|\eta^{\prime}\right|<1} \hat{S}\left(-k,-\tilde{\eta}^{\prime},-\eta^{\prime}\right) \hat{v}\left(k, \eta^{\prime}\right) k^{2} d^{2} \eta^{\prime}
$$

However, $\hat{S}\left(k, \eta^{\prime}, \tilde{\eta}^{\prime}\right)$ is defined by

$\delta\left(c_{0}(k-\tilde{k})\right) \hat{S}\left(k, \eta^{\prime}, \tilde{\eta}^{\prime}\right)=\int_{-\infty}^{\infty} \int_{-\infty}^{\infty} \iint e^{-i k \eta^{\prime} \cdot x^{\prime}} e^{i k c_{0} t} \mathcal{S}\left(t-\tau, x^{\prime}, y^{\prime}\right) e^{-i \tilde{k} c_{0} \tau} e^{i k \tilde{\eta}^{\prime} \cdot y^{\prime}} d^{2} x^{\prime} d^{2} y^{\prime} d \tau d t$

From this, we see that

$\delta\left(c_{0}(\tilde{k}-k)\right) \hat{S}\left(-k,-\eta^{\prime},-\tilde{\eta}^{\prime}\right)=\int_{-\infty}^{\infty} \int_{-\infty}^{\infty} \iint e^{-i k \eta^{\prime} \cdot x^{\prime}} e^{-i k c_{0} t} \mathcal{S}\left(t-\tau, x^{\prime}, y^{\prime}\right) e^{i \tilde{k} c_{0} \tau} e^{i k \tilde{\eta}^{\prime} \cdot y^{\prime}} d^{2} x^{\prime} d^{2} y^{\prime} d \tau d t$

Letting $t \rightarrow-t$ and $\tau \rightarrow-\tau$ in (55) shows that the kernel of (53) corresponds to the operator $T \mathcal{P S P} T$, given by

$$
(T \mathcal{P S P} T) V\left(t, x^{\prime}\right)=\int_{-\infty}^{\infty} \int_{-\infty}^{\infty} \mathcal{S}\left(\tau-t, x^{\prime}, y^{\prime}\right) V\left(t, y^{\prime}\right) d \tau d^{2} y^{\prime}
$$

Thus $(\mathcal{P S P})^{*}=T(\mathcal{P S P}) T$. 


\section{A.3 Compactness}

Theorem 1 Assume that the sound speed $c(x)$ is bounded and differs from $c_{0}$ only in a bounded subset of the half-space $x_{3} \leq-h<0$. Then the fixed-frequency scattering operator $\hat{S}$ is compact on the weighted space

$$
L_{g}^{2}=\left\{f:\left.\left.f\left(\eta^{\prime}\right)|1-| \eta^{\prime}\right|^{2}\right|^{1 / 4} \in L^{2}\right\}
$$

Proof. We use (47) and (12) to compute the square of the Hilbert-Schmidt norm of $\hat{S}$ in the space $L_{g}^{2}$ :

$$
\|\hat{S}\|_{H . S .}^{2}=\frac{k^{4}}{4} \iint\left|\int e^{-i k \eta \cdot y} V(y) \psi\left(k, y, \tilde{\eta}^{\prime}\right) d^{3} y\right|^{2}\left|\frac{1-\left|\tilde{\eta}^{\prime}\right|^{2}}{1-\left|\eta^{\prime}\right|^{2}}\right|^{1 / 2} d^{2} \eta^{\prime} d^{2} \tilde{\eta}^{\prime} .
$$

We use the fact that $\psi$ can be split into an incident and scattered field via (43). This allows us to split the kernel (47) into two parts:

$$
\hat{S}\left(k, \eta, \tilde{\eta}^{\prime}\right)=\hat{S}_{B}\left(k, \eta, \tilde{\eta}^{\prime}\right)+\hat{S}_{s c}\left(k, \eta, \tilde{\eta}^{\prime}\right)
$$

where the "Born" term is

$$
\hat{S}_{B}\left(k, \eta, \tilde{\eta}^{\prime}\right)=-\frac{i k}{2 \eta_{3}} \int e^{i k(\tilde{\eta}-\eta) \cdot y} V(y) d^{3} y
$$

and

$$
\hat{S}_{s c}\left(k, \eta, \tilde{\eta}^{\prime}\right)=-\frac{i k^{3}}{2 \eta_{3}} \int e^{-i k \eta \cdot y} V(y) \int g(y-z) V(z) \psi\left(k, z, \tilde{\eta}^{\prime}\right) d^{3} z d^{3} y .
$$

We compute the Hilbert-Schmidt norm of each part.

The Hilbert-Schmidt norm of the Born term is

$$
\left\|\hat{S}_{B}\right\|_{H . S .}^{2}=\frac{k^{2}}{4} \iint\left|\int e^{i k(\tilde{\eta}-\eta) \cdot y} V(y) d^{3} y\right|^{2}\left|\frac{1-\left|\tilde{\eta}^{\prime}\right|^{2}}{1-\left|\eta^{\prime}\right|^{2}}\right|^{1 / 2} d^{2} \eta^{\prime} d^{2} \tilde{\eta}^{\prime} .
$$

The $y$ integral of (62) can be written

$$
\int e^{i k\left(\tilde{\eta}^{\prime}-\eta^{\prime}\right) \cdot y^{\prime}} e^{-i k\left(\tilde{\eta}_{3}+\eta_{3}\right) y_{3}} V(y) d^{3} y
$$

For $\left|\eta^{\prime}\right|<1$, for which $\eta_{3}$ is real, this integral is bounded when $V$ is integrable. For $\left|\eta^{\prime}\right|>1$, the integral decays exponentially because $V$ is supported in the region where $y_{3} \leq h<0$. The same comments apply to the behavior in $\tilde{\eta}^{\prime}$. Thus this integral is bounded by $c \exp \left(-h k\left(\left|\eta^{\prime}\right|+\left|\tilde{\eta}^{\prime}\right|\right)\right)$. This estimate can easily be used to show that $\left\|\hat{S}_{B}\right\|_{H . S .}$ is finite. 
The Hilbert-Schmidt norm of the scattered part is

$$
\left\|\hat{S}_{s c}\right\|_{H . S .}^{2}=\frac{k^{6}}{4} \iint\left|\int e^{-i k \eta \cdot y} V(y) \int g(y-z) V(z) \psi\left(k, z, \tilde{\eta}^{\prime}\right) d^{3} z d^{3} y\right|^{2}\left|\frac{1-\left|\tilde{\eta}^{\prime}\right|^{2}}{1-\left|\eta^{\prime}\right|^{2}}\right|^{1 / 2} d^{2} \eta^{\prime} d^{2} \tilde{\eta}^{\prime} .
$$

An application of the Cauchy-Schwarz inequality shows that the $y$ integral of (64) is bounded by

$$
\left\|\left.e^{-i k \eta \cdot y} V(y)\right|^{2}\right\|_{L^{2}(y)}\left\||V(y)|^{1 / 2} \int g(y-z) V(z) \psi\left(k, z, \eta^{\prime}\right) d^{3} z\right\|_{L^{2}(y)}
$$

Direct computation shows that the first norm in (65) is bounded by $c \exp \left(-h k\left|\eta^{\prime}\right|\right)$. Standard scattering theory arguments (See Appendix C) can be used to show that the second norm appearing in (65) is bounded by $c \exp \left(-h k\left|\tilde{\eta}^{\prime}\right|\right)$; thus the $y$ integral satisfies the same bounds as in the Born term. QED

Remark. In [2], it was shown that on $L_{g}^{2}$, the operator $P \hat{S} P$ has norm less than or equal to one.

For future reference, we note that the operator $a(k)=(P S P)^{*}(P S P)$ is given explicitly as

$$
(F a f)\left(\tilde{\eta}^{\prime}\right)=\int_{\left|\tilde{\eta}^{\prime}\right|<1} \int_{\left|\zeta^{\prime}\right|<1} \hat{S}\left(-k,-\tilde{\eta}^{\prime},-\zeta^{\prime}\right) \hat{S}\left(k, \zeta^{\prime}, \eta^{\prime}\right) d^{2} \zeta^{\prime} \hat{f}\left(\eta^{\prime}\right) k^{4} d^{2} \eta^{\prime}
$$

\section{B Appendix: Convergence of the Iterative Algo- rithm}

For simplicity of notation we consider only the case when $\mathcal{S}_{0}=0$. In this case, the $n$th iterate is

$$
U_{n}^{\downarrow}=\frac{(T(\mathcal{P S P}) T(\mathcal{P S P}))^{n} U_{0}^{\downarrow}}{\left((T(\mathcal{P S P}) T(\mathcal{P S P}))^{n} U_{0}^{\downarrow}, \Psi_{B}\right)_{W}}=\frac{\mathcal{A}^{n} U_{0}^{\downarrow}}{\left(\mathcal{A}^{n} U_{0}^{\downarrow}, \Psi_{B}\right)_{W}},
$$

where $\mathcal{A}=(\mathcal{P S P})^{*}(\mathcal{P S P})$, where the star denotes the adjoint with respect to the flux inner product $(\cdot, \cdot)_{W}$. We simplify the notation by dropping the arrow on $U$. Because we expect the limit to be a distribution, we consider the quantity $\left(U_{n}, \Phi\right)_{W}=\int\left(u_{n}, \phi\right)_{x^{\prime}} c_{0}^{2} k^{4} d k$, where $\Phi$ is a smooth test function, $\phi=\mathcal{F} \Phi$, and $(,)_{x^{\prime}}$ denotes the weighted inner product that for smooth functions is $(f, \phi)_{x^{\prime}}=(\hat{f}, \hat{\phi})_{L_{g}^{2}}=$ $\int \hat{f}\left(\eta^{\prime}\right) \overline{\hat{\phi}\left(\eta^{\prime}\right)}\left|\eta_{3}\right| d^{2} \eta^{\prime}$.

Specifically, we consider test function that are functions of $t$ and $x^{\prime}$. When inverse Fourier transformed in $t$ and Fourier transformed in space, at each frequency they 
must be in $L_{g}^{2}$, and in the frequency variable they must be integrable and (uniformly) supported in the compact interval $[-B, B]$. We denote this space of test functions by $X$.

In the frequency domain, the $n$th iteration is $\mathcal{F}^{-1}\left(\mathcal{A}^{n} U\right)=a^{n} u$, where $\mathcal{F}^{-1}$ denotes the inverse Fourier transform (6) and $a=\mathcal{F}^{-1} \mathcal{A} \mathcal{F}=(P S P)^{*}(P S P)$. From our normalization of $U_{n}$, we have

$$
\left(U_{n}, \Phi_{B}\right)_{W}=\frac{\left(\mathcal{A}^{n} U, \Phi_{B}\right)_{W}}{\left(\mathcal{A}^{n} U, \Psi_{B}\right)_{W}}=\frac{\int\left\langle a^{n} u, \phi_{B}\right\rangle_{x^{\prime}} c_{0}^{2} k^{4} d k}{\int\left\langle a^{n} u, \psi_{B}\right\rangle_{x^{\prime}} c_{0}^{2} k^{4} d k} .
$$

We note that $\mathcal{A}$ and $a(k)$ are self-adjoint on the space $W$ and on $L_{g}^{2}$, respectively. Moreover, Theorem 1 shows that $a(k)$ is compact on $L_{g}^{2}$, and it can therefore be written $a(k)=\sum_{l} \lambda_{l}(k) P_{l}(k)$, where $\lambda_{l} \geq \lambda_{l+1}$ and the $P_{l}$ are orthogonal projections. Because $\mathcal{A}$ is non-negative, all the eigenvalues $\lambda_{l}$ are non-negative. We see from (66) that $a$ is analytic in $k$, and the $\lambda_{\mathrm{s}}$ are therefore piecewise analytic [8].

Suppose that $\lambda_{0}(k)$ attains its maximum in the set $\{|k|<B\}$ at $k_{0}$, and that $\lambda(k)=M$. Then in a neighborhood of $k_{0}, \lambda(k)$ has a Taylor expansion whose first two terms are $M-b\left(k-k_{0}\right)^{p}$ for some $b$ and some integer $p$. We call $p$ the order of $\lambda_{0}$.

We allow eigenvalues with different indices to coincide at a point; thus it is possible that a finite number of eigenvalues also attain the maximum $M$ at $k_{0}$. In this case, these eigenvalues have a Taylor expansion similar to that of $\lambda_{0}$, possibly with different $b$ s and $p$ s. In this case we also refer to the relevant $p$ as the order of the eigenfunction.

We will need the following lemma.

Lemma 1 Assume that $b$ is positive and that $p$ is an integer. Then for large $n$,

$$
I(n, p)=\int_{0}^{h}\left(1-b k^{p}\right)^{n} d k \sim \frac{C(p)}{(b n)^{1 / p}},
$$

where $C(p)$ is a nonzero constant independent of $n$. Thus the convergence to zero of $I(n, p)$ is slower for larger $p$ and smaller $b$.

Proof. Let $s=b^{1 / p} k$. Then $I=b^{-1 / p} \int_{0}^{b^{1 / p} h}\left(1-s^{p}\right)^{n} d s$. Replacing the upper limit by 1 results in an error that is exponentially small in $n$. Denote by $I_{n}$ the integral $\int_{0}^{1}\left(1-s^{p}\right)^{n} d s$. Then we can write

$$
I_{n+1}=\int_{0}^{1}\left(1-s^{p}\right)\left(1-s^{p}\right)^{n} d s=I_{n}-\int_{0}^{1} s^{p}\left(1-s^{p}\right)^{n} d s .
$$

In the integral of $(\sqrt{70})$, we integrate by parts, differentiating $s$ and integrating $s^{p-1}(1-$ $\left.s^{p}\right)^{n}$. The boundary term vanishes, and (70) becomes

$$
I_{n+1}=I_{n}+\frac{1}{p(n+1)} I_{n+1} \text {. }
$$


Solving for $I_{n+1}$ gives the recursion

$$
I_{n+1}=\frac{p(n+1)}{p(n+1)+1} I_{n} .
$$

Since $I_{0}=1$, we have

$$
I_{n}=\left(\frac{p}{p+1}\right)\left(\frac{2 p}{2 p+1}\right) \cdots\left(\frac{n p}{n p+1}\right) .
$$

Taking reciprocals and logs and expanding, we find that

$$
\begin{aligned}
-\log I_{n} & =\sum_{j=1}^{n} \log (1+1 /(j p)) \\
& =\frac{1}{p} \sum_{j=1}^{n} \frac{1}{j}-\sum_{m=2}^{\infty} \frac{(-1)^{m}}{m p^{m}} \sum_{j=1}^{n} \frac{1}{k j^{m}}
\end{aligned}
$$

Exponentiating and taking reciprocals again, we have

$$
I_{n}=C(n, p) \exp \left(-\frac{1}{p} \sum_{j=1}^{n} \frac{1}{j}\right)
$$

where $C(n, p)$ has the large-n limit

$$
\lim _{n \rightarrow \infty} C(n, p)=\exp \left(-\sum_{m=2}^{\infty} \frac{(-1)^{m}}{m p^{m}} \zeta(m)\right)
$$

where $\zeta(m)$ denotes the Riemann-zeta function $\zeta(m)=\sum_{k=1}^{\infty} k^{-m}$. Thus we see that the large-n behavior of $I_{n}$ is determined by the second factor of (75).

We determine the large-n behavior of this second factor as follows. From approximating the sum by a Riemann integral, we have the estimate

$$
\log n \leq \log (n+1) \leq \sum_{j=1}^{n} \frac{1}{j} \leq 1+\log n
$$

We multiply by $-1 / p$ and exponentiate to obtain

$$
e^{-1 / p} n^{-1 / p} \leq \exp \left(-\frac{1}{p} \sum_{j=1}^{n} \frac{1}{j}\right) \leq n^{-1 / p}
$$

QED 
Theorem 2 Assume that $a$ is an analytic self-adjoint-compact-operator-valued function of $k$ having the representation $a(k)=\sum_{l} \lambda_{l}(k) P_{l}(k)$, where $\lambda_{l} \geq \lambda_{l+1}$ and the $P_{l}$ are orthogonal projections. Assume that $\lambda_{0}$ is not a constant function of $k$. Then for test functions $\phi_{B}\left(k, x^{\prime}\right)$ and $\psi_{B}\left(k, x^{\prime}\right)$ in $X$ whose support in the frequency domain is in the set $\{|k| \leq B\}$,

$$
\lim _{n \rightarrow \infty} \frac{\int\left(a^{n} u, \phi_{B}\right)_{x^{\prime}} k d k}{\int\left(a^{n} u, \psi_{B}\right)_{x^{\prime}} k d k}=\frac{\sum_{l, j} \beta_{l j}\left(P_{l} u, \phi_{B}\right)_{x^{\prime}}\left(k_{j}\right)}{\sum_{l, j} \beta_{l j}\left(P_{l} u, \psi_{B}\right)_{x^{\prime}}\left(k_{j}\right)}
$$

where the sums are over those indices $j$ and $l$ for which $\lambda_{l}\left(k_{j}\right)=M$, where $M$ is the maximum of $\lambda_{0}$ in the set $\{k:|k| \leq B\}$, and for which $\lambda_{l}$ has maximal order at $k_{j}$.

Proof

The representation for $a$ allows us to write (68) as

$$
\left(U_{n}, \Phi_{B}\right)_{W}=\frac{\int \sum_{l} \lambda_{l}^{n}\left(P_{l} u, \phi_{B}\right)_{x^{\prime}} k d k}{\int \sum_{l} \lambda_{l}^{n}\left(P_{l} u, \psi_{B}\right)_{x^{\prime}} k d k}
$$

The $\lambda_{l}$ and $P_{l}$ are peicewise analytic functions of $k$ [8]. In particular $\lambda_{0}(k)$ is piecewise analytic, and therefore attains its maximum $M$ on a discrete subset of the set $\{|k| \leq$ $B$ \}. We cover the support of $\phi$ with open intervals $N_{j}$ so that each $N_{j}$ contains only one $k_{j}$. We decompose the test function $\phi_{B}$ as $\phi_{B}=\sum_{j} \phi_{j}$ [5], where the $\phi_{j}$ are in $C_{0}^{\infty}\left(N_{j}\right)$ and $\phi_{j}=\phi_{B}$ in a neighborhood of $k_{j}$. We carry out a similar decomposition for $\psi_{B}$.

With the notation $f_{l, j}(k)=k\left(P_{l} u, \phi_{j}\right)_{x^{\prime}}$ and $g_{l, j}(k)=k\left(P_{l} u, \psi_{j}\right)_{x^{\prime}}$, we can write (80) as

$$
\left(U_{n}, \Phi_{B}\right)_{W}=\frac{\sum_{l, j} \int_{N_{j}} \lambda_{l}^{n}(k) f_{l, j}(k) d k}{\sum_{l, j} \int_{N_{j}} \lambda_{l}^{n}(k) g_{l, j}(k) d k} .
$$

We divide the numerator and denominator of (81) by $M^{n}$, and write $r_{l}(k)=$ $\lambda_{l}(k) / M$; thus $\left|r_{0}\right| \leq 1$, and $\left|r_{l}\right|<1$ for all but a finite number of values of $l$. Then (81) can be written

$$
\left(U_{n}, \Phi_{B}\right)_{W}=\frac{\sum_{l, j} \int_{N_{j}} r_{l}^{n}(k) f_{l, j}(k) d k}{\sum_{l, j} \int_{N_{j}} r_{l}^{n}(k) g_{l, j}(k) d k}
$$

We write

$$
I_{l, j}^{n}=\int_{N_{j}} r_{l}^{n}(k) f_{l, j}(k) d k
$$

We multiply and divide $I_{l, j}^{n}$ by $\int_{N_{j}} r_{0}^{n}(k) d k$, and write $\zeta_{l}^{n}=r_{l}^{n} / \int_{N_{j}} r_{0}^{n} d k$.

For those $l=0,1, \ldots L_{j}$ for which $\lambda_{l}$ attains the maximum $M$ and thus $r_{l}$ attains the value 1 , we add and subtract $f_{l, j}\left(k_{j}\right)$ to the quotient, obtaining

$$
I_{l, j}^{n}=\left(f_{l, j}\left(k_{j}\right)+\int_{N_{j}} \zeta_{l}^{n}(k)\left(f_{l, j}(k)-f_{l, j}\left(k_{j}\right)\right) d k\right) \int_{N_{j}} r_{0}^{n}(k) d k
$$


We will show that the integral term within the parentheses on the right side of (84) vanishes as $n$ goes to infinity. For this we use the two facts that 1) except at $k=k_{j}$, $\zeta_{l}^{n}$ converges to zero pointwise as $n$ goes to infinity; and 2) $\int \zeta_{l}^{n}=1$ for all $n$.

Given $\epsilon>0$, we choose $N_{j}^{\epsilon}$ so small that on $N_{j}^{\epsilon},\left|f_{l, j}(k)-f_{l, j}\left(k_{j}\right)\right|<\epsilon / 2$. The integral in parentheses on the right side of (84) we split into two integrals, namely $A^{n}$ and $B^{n}$, where

$$
A^{n}=\int_{N_{j}^{\epsilon}} \zeta_{l}^{n}(k)\left(f_{l, j}(k)-f_{l, j}\left(k_{j}\right)\right) d k,
$$

and

$$
B^{n}=\int_{N_{j} \backslash N_{j}^{\epsilon}} \zeta_{l}^{n}(k)\left(f_{l, j}(k)-f_{l, j}\left(k_{j}\right)\right) d k .
$$

Then $A^{n}<\epsilon / 2$. Next, we choose $N$ so large that for $n$ greater than $N, B^{n}<\epsilon / 2$. This shows that the integral in parentheses on the right side of (84) vanishes as $n$ goes to infinity. Thus (84) is a product of a factor converging to $f_{l, j}\left(k_{j}\right)$ and a factor converging to zero.

For $l=L_{j}+1, L_{j}+2, \ldots$, for which $\lambda_{l}$ is strictly less than $M$, we write

$$
I_{l, j}^{n}=\left(\int \zeta_{l}^{n}(k) f_{l, j}(k) d k\right) \int_{N_{j}} r_{0}^{n}(k) d k .
$$

In this case, $\zeta_{l}^{n}$ converges to zero pointwise for all $k$. Thus the integral in parentheses of $I_{l, j}^{n}$ converges to zero by the Lebesgue Dominated Convergence Theorem.

To estimate the tail of the sum over $l$ in (82), we choose $l_{0}$ so large that for $l>l_{0}$, $\zeta_{l}(k)<1 / 2$ for all $k$ in $N_{j}$. This is possible because the the compactness of $a$ implies that its eigenvalues decrease to zero. Thus for each $j$ we have

$$
\left|\sum_{l>l_{0}} I_{l, j}^{n}\right|=\left|\sum_{l>l_{0}} \int_{N_{j}} \zeta_{l}^{n}(k)\left(P_{l} u, \phi_{j}\right)_{x^{\prime}} k d k\right| \leq \frac{1}{2^{n}} \int_{N_{j}} \sum_{l>l_{0}}\left|\left(u, \varphi_{l}\right)_{x^{\prime}}\left(\varphi_{l}, \phi_{j}\right)_{x^{\prime}} k\right| d k,
$$

where the $\varphi_{l}$ are the normalized eigenfunctions of $a$. Here it may be necessary to reindex the sum. Each of the sequences $\left(u, \varphi_{l}\right)_{x^{\prime}}$ and $\left(\varphi_{l}, \phi_{j}\right)_{x^{\prime}}$ is in $l^{2}$, and the inner product of two $l^{2}$ sequences is in $l^{1}$. We therefore find that the sum over $l$ is bounded by $\|u\|_{k}\left\|\phi_{j}\right\|_{k}$, where $\|\cdot\|_{k}$ denotes the norm in the space $L_{k}^{2}$. Thus we have

$$
\left|\sum_{l>l_{0}} I_{l, j}^{n}\right| \leq \frac{1}{2^{n}} \int_{N_{j}}\|u\|_{k}\left\|\phi_{j}\right\|_{k} k d k
$$

which shows that the tail of the sum converges to zero as $n$ goes to infinity.

The same arguments, of course, apply to the denominator of (82). Thus we see that the leading order behavior of (82) is given by the expression

$$
\left(U_{n}, \Phi_{B}\right)_{W} \sim \frac{\sum_{l, j} f_{l, j}\left(k_{j}\right) \int_{N_{j}} r_{0}^{n}(k) d k}{\sum_{l, j} g_{l, j}\left(k_{j}\right) \int_{N_{j}} r_{0}^{n}(k) d k},
$$


where the sum in $l$ is over those values for which $\lambda_{l}$ attains the maximum $M$ at $k_{j}$. The terms $\int_{N_{j}} r_{0}^{n}(k) d k$, however, go to zero for large $n$. We must therefore consider their behavior in more detail.

As we have seen, in the neighborhood of $k=k_{j}, r_{0}(k)$ has an expansion of the form $r_{0}(k)=1-b_{j}\left(k-k_{j}\right)^{p_{j}}+\ldots$, where the positive integer $p_{j}$ is the order of the eigenfunction at $k_{j}$. The lemma shows that the order $p_{j}$ controls the speed with which $\int_{N_{j}} r_{0}^{n}(k) d k$ goes to zero with $n$ : the larger $p_{j}$, the more slowly $\int r_{0}^{N} d k$ coverges to zero. We divide the numerator and denominator of (82) by $\int_{N_{j}} r_{0}^{n} d k$ corresponding to the slowest decay. Finally, we take the limit of the resulting quotient as $n$ goes to infinity. This shows that the quotient (82) converges to

$\left(U_{n}, \Phi_{B}\right)_{W} \sim \frac{\sum_{l, j} \beta_{j}\left(P_{l} u, \phi\right)_{x^{\prime}}\left(k_{j}\right)}{\sum_{l, j} \beta_{j}\left(P_{l} u, \psi\right)_{x^{\prime}}\left(k_{j}\right)}=\iint \sum_{l, j} \beta_{j} \frac{P_{l} u\left(k_{j}, x^{\prime}\right)}{\sum_{l, i} \beta_{i}\left(P_{l} u, \psi\right)_{x^{\prime}}\left(k_{i}\right)} \delta_{k_{j}}(k) \phi\left(k, x^{\prime}\right) d^{2} x^{\prime} d k$

where the $\beta_{j}$ are proportional to $\beta_{j}=k_{j} C\left(p_{j}\right) / b_{j}^{1 / p_{j}}$, and where the sums are over those indices $j$ and $l$ for which $\lambda_{l}$ has maximal order at $k_{j}$.

QED.

Corollary 1 Assume that the sound speed $c(x)$ is bounded and differs from $c_{0}$ only in a bounded subset of the half-space $x_{3} \leq-h<0$. Then for test functions $\Psi_{B}, \Phi_{B}$ in $X$ whose Fourier transforms with respect to time are supported in $-B \leq k \leq B$, $U_{n}$ as defined by (67) converges to

$$
\frac{1}{2 \pi \sum_{l, i} \beta_{k}\left(P_{l} u, \psi_{B}\right)_{x^{\prime}}\left(k_{i}\right)} \sum_{l, j} \beta_{j} P_{l} u\left(k_{j}, x^{\prime}\right) e^{-i k_{j} c_{0} t}
$$

where the sums are over those indices $j$ and $l$ for which $\lambda_{l}\left(k_{j}\right)$ attains the maximum $M$ and has maximal order.

Proof. To apply Theorem 2, we need only check that $a=\mathcal{F}^{-1}(\mathcal{P} \mathcal{S P})^{*}(\mathcal{P S P}) \mathcal{F}$ is indeed an analytic compact-operator-valued function of $k$. Analyticity was shown in section A.1; compactness was shown in section A.3. The largest eigenvalue $\lambda_{0}$ cannot be constant: $S$ is zero at $k=0$, which implies that all the $\lambda_{j}$ are zero there. Thus if $\lambda_{0}$ were constant it would be zero, and $S$ itself would be zero.

We note that both the numerator and denominator in (92) can be zero, in which case (92) is not defined. However we do not study this case since the denominator is non-zero for a generic test function $\Psi_{B}$. QED 


\section{Appendix: Results from "Standard" Scattering Theory}

The solution of the equation

$$
\left(\nabla^{2}+k^{2}-V(x)\right) \psi(k, x)=0
$$

corresponding to an incident plane wave and a scattered field satisfying outgoing boundary conditions satisfies the Lippmann-Schwinger integral equation

$$
\psi\left(k, x, \tilde{\eta}^{\prime}\right)=\exp (i k \tilde{\eta} \cdot x)-k^{2} \int g(k, x, y) V(y) \psi\left(k, y, \tilde{\eta}^{\prime}\right) d^{3} y,
$$

where $g$ is the usual outgoing Green's function (44).

The initial difficulty with the Lippmann-Schwinger equation is that the incident field has infinite energy. This difficulty, however, can be circumvented by multiplying the whole equation by $|V(x)|^{1 / 2} \llbracket 18$. This converts (94) into

$$
\zeta\left(k, x, \tilde{\eta}^{\prime}\right)=\zeta_{0}\left(k, x, \tilde{\eta}^{\prime}\right)+k^{2} \int K(k, x-y) \zeta\left(k, y, \tilde{\eta}^{\prime}\right) d^{3} y,
$$

where

$$
\begin{aligned}
\zeta\left(k, x, \tilde{\eta}^{\prime}\right) & =|V(x)|^{1 / 2} \psi\left(k, x, \tilde{\eta}^{\prime}\right), \\
\zeta_{0}\left(k, x, \tilde{\eta}^{\prime}\right) & =|V(x)|^{1 / 2} e^{i k \tilde{\eta} \cdot x} \\
V_{1 / 2}(y) & =V(y) /|V(y)|^{1 / 2} \\
K(x, y) & =|V(x)|^{1 / 2} g(k, x, y) V_{1 / 2}(y)
\end{aligned}
$$

When $V$ has compact support, (95) is an integral equation on a bounded region. The kernel $K$ is an Hilbert-Schmidt-valued function that is analytic in the entire complex $k$-plane. By the analytic Fredholm theorem [16], the integral equation (95) is therefore uniquely solvable everywhere except at a discrete set of values of $k$, (the "exceptional points") and moreover the solution $\zeta$ is a meromorphic function of $k$ with poles at these exceptional points. In addition, the arguments of [1] [17] show that the only possible real exceptional point is $k=0$. However, when $k=0$, we also have $k=0$, and (95) reduces to the equation $\zeta=|V|^{1 / 2}$. Thus the operator $\left(I-k^{2} K\right)^{-1}$ is analytic in a neighborhood of the real $k$-axis.

This argument shows that for each $k, \zeta=|V|^{1 / 2} \psi$ is in $L^{2}$. Then the quantity needed in section A.3, namely $\left\||V|^{1 / 2} \int g V \psi\right\|$, can be rewritten as $\|K \zeta\|=\| K(I-$ $K)^{-1} \zeta_{0}\|\leq\| K\|\|(I-K)^{-1}\|\| \zeta_{0} \|$. Moreover, by explicit computation, we see that $\left\|\zeta_{0}\left(k, \cdot, \tilde{\eta}^{\prime}\right)\right\| \leq c e^{-h k\left|\tilde{\eta}^{\prime}\right|}$, where $V$ is supported in the region $y_{3} \leq-h<0$. 


\section{References}

[1] S. Agmon, "Spectral properties of Schrödinger operators and scattering theory", Ann. Scuola Norm. Sup. Pisa Cl. Sci. II, 2 (1975), 151-218.

[2] M. Cheney and D. Isaacson, "Inverse Problems for a Perturbed Dissipative HalfSpace", Inverse Problems 11 (1995) 865-888.

[3] E. Cherkaeva and A.C. Tripp, "On optimal design of transient electromagnetic waveforms", SEG97 Expanded Abstracts, 67th Annual Meeting of Soc. Exploration Geophys. (1997) 438-441.

[4] J.W. Dettman, Applied Complex Variables, Dover, New York, 1965.

[5] L. Hörmander, The Analysis of Linear Partial Differential Operators I, Springer, New York, 1983.

[6] D. Isaacson, "Distinguishability of conductivities by electric current computed tomography", IEEE Trans. on Medical Imaging MI-5(2):92-95, 1986.

[7] G. Eskin and J. Ralston, "Inverse coefficient problems in perturbed half spaces", Inverse Problems 15 (1999) 683-699.

[8] T. Kato, Perturbation Theory for Linear Operators, Springer-Verlag, Berlin, 1966.

[9] M.S. Lehtinen, On statistical inversion theory. Theory and applications of inverse problems, Pitman Res. Notes Math. Ser. 167, 46-57, 1988.

[10] M.S. Lehtinen, L. Päivärinta, and E. Somersalo, "Linear inverse problems for generalised random variables", Inverse Problems 5 (1989), no. 4, 599-612.

[11] M. Lassas, M. Cheney, and G. Uhlmann, "Uniqueness for a wave propagation inverse problem in a half space", Inverse Problems 14, 679-684 (1998) .

[12] T.D. Mast, A.I. Nachman, and R. C. Waag, "Focusing and imaging using eigenfunctions of the scattering operator", J. Acoust. Soc. Am. 102, Pt. 1 (1997) 715-725.

[13] R.G. Newton, Inverse Schrödinger Scattering in Three Dimensions, Springer, New York, 1989.

[14] C. Prada and M. Fink, "Eigenmodes of the time reversal operator: A solution to selective focusing in multiple-target media", Wave Motion 20 (1994), 151-163.

[15] C. Prada, J.-L. Thomas, and M. Fink, "The iterative time reversal process: Analysis of the convergence", J. Acoust. Soc. Am. 97 (1995) 62-71. 
[16] M. Reed and B. Simon, Methods of Modern Mathematical Physics. I. Functional Analysis, Academic Press, New York, 1972.

[17] M. Reed and B. Simon, Methods of Modern Mathematical Physics. IV. Functional Anlaysis, Academic Press, New York, 1978.

[18] B. Simon, Quantum Mechanics for Hamiltonians Defined as Quadratic Forms, Princeton University Press, New Jersey, 1971.

[19] R. Weder, Spectral and Scattering Theory for Wave Propagation in Perturbed Stratified Media", Springer, New York, 1991.

[20] Y. Xu, "Reciprocity relations and completeness of far-field pattern vectors for obstacle scattering of acoustic wave in a stratified medium", Math. Methods in the Applied Sciences 18 (1995) 41-66. 